\title{
Basic leucine zipper transcription factor SlbZIP1 mediates salt and drought stress tolerance in tomato
}

\author{
Mingku Zhu ${ }^{1,2^{*}+}$, Xiaoqing Meng ${ }^{1,2+}$, Jing Cai ${ }^{1,2}, \mathrm{Ge} \mathrm{Li}^{1,2}$, Tingting Dong ${ }^{1,2}$ and Zongyun $\mathrm{Li}^{1,2^{*}}$
}

\begin{abstract}
Background: Basic region/leucine zipper (bZIP) transcription factors perform as crucial regulators in ABA-mediated stress response in plants. Nevertheless, the functions for most bZIP family members in tomato remain to be deciphered.

Results: Here we examined the functional characterization of SIbZIP1 under salt and drought stresses in tomato. Silencing of SIbZIP1 in tomato resulted in reduced expression of multiple ABA biosynthesis- and signal transduction-related genes in transgenic plants. In stress assays, SIbZIP1-RNAi transgenic plants exhibited reduced tolerance to salt and drought stresses compared with WT plants, as are evaluated by multiple physiological parameters associated with stress responses, such as decreased ABA, chlorophyll contents and CAT activity, and increased MDA content. In addition, RNA-seq analysis of transgenic plants revealed that the transcription levels of multiple genes encoding defense proteins related to responses to abiotic stress (e.g. endochitinase, peroxidases, and lipid transfer proteins) and biotic stress (e.g. pathogenesis-related proteins) were downregulated in SIbZIP1-RNAi plants, suggesting that SIbZIP1 plays a role in regulating the genes related to biotic and abiotic stress response.
\end{abstract}

Conclusions: Collectively, the data suggest that SIbZIP1 exerts an essential role in salt and drought stress tolerance through modulating an ABA-mediated pathway, and SIbZIP1 may hold potential applications in the engineering of salt- and drought-tolerant tomato cultivars.

Keywords: Abscisic acid, Drought stress, Salt stress, SIbZIP1, Tomato

\section{Background}

Drought, salt and extreme temperatures are primary environmental factors that limit plant growth and reduce crop production [1]. Nevertheless, some plant species have developed diverse and complicated mechanisms at molecular, cellular and physiological levels that enable them to endure these unfavorable situations. Transcription factors (TFs) are pivotal regulatory proteins in signal transduction networks activated in plants response to various stresses [2]. Numerous studies have shown that plenty of such regulatory mechanisms generally

\footnotetext{
*Correspondence: mingkuzhu007@126.com; zongyunli@jsnu.edu.cn ${ }^{\dagger}$ Equal contributors

${ }^{1}$ School of Life Sciences, Jiangsu Normal University, 101 Shanghai Road, Xuzhou, Jiangsu Province 221116, People's Republic of China

Full list of author information is available at the end of the article
}

follow the phytohormone ABA (abscisic acid)-dependent pathway. ABA has usually been deemed as the global plant stress hormone because of its remarkable effects on growth regulation during stress responses [3].

TFs often bind to specific cis-elements in the promoters of many genes to regulate their expression thereby enhancing stress tolerance [4]. Among numerous TF families, the bZIP TF family, known as ABA-response element binding factors (AREB) or ABF (ABRE-binding factors) has been isolated [5]. bZIP proteins are bipartite in structure containing DNA binding domains consisting of abundant basic amino acids that bind to DNA, and leucine zippers characterized by leucine residues spaced regularly at seven amino acid intervals [6]. Correlative research has indicated that ABA-dependent bZIP TFs generally bind to a conserved sequence with an ACGT core cis-element, 
especially A-box (TACGTA), ABRE (ACGTGG/TC), Cbox (GACGTC) and G-box (CACGTG) [7, 8].

In plants, extensive documents have shown that bZIP genes participate in plenty of biological processes, including seed maturation, unfolded protein response, photomorphogenesis and light signaling, hormone signaling and organ development [9]. Furthermore, bZIP proteins also perform as crucial components in the signal transduction networks that mediate the response to various stresses, including salt, drought, cold and pathogen defense. Presently, bZIPs are well characterized in the model plants Arabidopsis and rice, and a total of 75 and 109 bZIPs have been identified, respectively [6, 10]. In Arabidopsis, the bZIP proteins AREB1, AREB2 and ABF3 cooperatively regulate the ABREdependent ABA signaling that is involved in drought resistance [11]. Overexpression of Arabidopsis bZIP gene AtTGA4 confers enhanced drought resistance by improving nitrate transport and assimilation [12]. Contrarily, silencing of AtbZIP24 using RNAi enhances salt tolerance in Arabidopsis [13]. In rice, qRT-PCR analysis showed that the transcription of most OsbZIP genes is upregulated by ABA and abiotic stress. Overexpression of OsbZIP72 in rice displays a hypersensitivity to $A B A$, enhanced expression levels of ABA-responsive genes and improves drought resistance [14]. Overexpression of OsbZIP23 and OsbZIP71 enhances remarkably salt and drought tolerance through an ABAdependent pathway in rice $[15,16]$. Furthermore, overexpression of wheat ABI-like ( $A B L$ ) bZIP TF gene TaABL1 confers multiple stress tolerance against salt, drought and cold stresses [17]. Overexpression of GhABF2 evidently enhances salt and drought tolerance both in Arabidopsis and cotton, while silencing $G h A B F 2$ increases the sensibility to PEG and salt stresses [18].

Tomato has been one of the most widely produced crops worldwide because of its great nutritive and commercial values, and also one of the best-characterized model plants employed in genetic studies. However, for most tomato cultivars, salt produces adverse effects on tomato growth and development, such as decreased seed germination, inhibited growth and reduced fruit productivity [19]. Recently, the expression of 69 tomato SlbZIPs in various tissues, and the response to hormone and biotic stress have been reported [20]. However, the functions of most tomato bZIP genes in terms of the abiotic stress response remain unclear. The best-studied tomato bZIP gene, SlAREB1, is shown to play crucial functions in salt and drought tolerance. Meanwhile, it may act as a link of ABA signaling transduction to biotic stress responses [21-23]. Expression of several tomato bZIP genes such as LebZIP2, FD, SlAREB2 and ABZ1 is shown to be upregulated by salt, drought and wounding stresses or by organ-specific signals [22, 24-26]. Collectively, despite the involvement of bZIP TFs in various biological processes, the specific molecular functions of most tomato bZIP genes remain to be clarified.

Herein, we reported the functional characterization of SlbZIP1 (Solyc01g079480.3, Genbank accession No. AF176641, formerly known as LebZIP1), it was then renamed as SlbZIPO4 and fell into Group A of bZIP TFs based on the systematic analysis of the bZIP TFs in tomato. In this study, we conducted a further functional analysis by silencing SlbZIP1 gene in tomato to investigate its roles in salt and drought tolerance.

\section{Results}

Expression profiles of SIbZIP1 in WT tomato and ripening mutant fruits

The tissue-specific expression of SlbZIP1 in WT tomato was quantified by qRT-PCR in various tissues. The results exhibited that SlbZIP1 expression was relatively high in roots, mature leaves and sepals, but moderate to weak signals were observed in stems, flowers and fruits (Fig. 1a). To investigate whether there was any
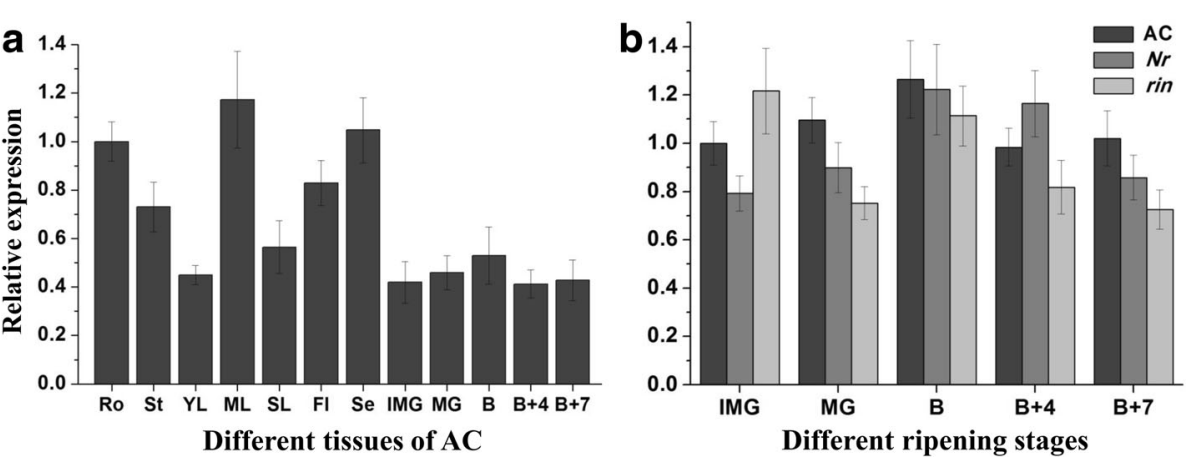

Fig. 1 Expression profiles of SIbZIP1 gene in different tissues of WT and ripening mutant fruits. a Expression patterns of SIbZIP1 in different tissues of WT plants. The relative expression levels were normalized to 1 in roots. $\mathbf{b}$ Expression patterns of SIbZIP1 between WT and ripening mutant fruits. Total RNA from the $\mathrm{Nr}$ (never ripe) and rin (ripening inhibitor) fruits from IMG to B + 7 equivalent stages with WT was subjected to qRT-PCR. The relative expression levels were normalized to 1 in IMG fruits of AC. Ro, roots; St, stems; Yl, young leaves; MI, mature leaves; $\mathrm{Sl}$, senescent leaves; FI, flowers; Se, sepals; IMG, immature green; MG, mature green; B, breaker; B + 4, $4 \mathrm{~d}$ after breaker stage; B + 7, $7 \mathrm{~d}$ after breaker stage. Bars represent the mean of three biological replicates \pm SE 
relationship between SlbZIP1 and tomato fruit ripening mutants, SlbZIP1 expression in rin and $N r$ mutants was also checked from IMG to $B+7$ stage (equivalent stages with AC fruits). No distinct difference was detected among rin, $\mathrm{Nr}$ and WT fruits (Fig. 1b), suggesting that the expression of SlbZIP1 is not affected by the single locus RIN and $N r$.

SIbZIP1 expression was induced by multiple abiotic stress and hormone treatments

Although previous research has shown that SlbZIP1 expression is upregulated by wounding and cold stresses, and ABA prevents its rapid wound-induced expression [27], the detailed response profiles of
SlbZIP1 gene to hormone and abiotic stress treatments have not been reported. Then a further expression analysis was carried out to speculate on its physiological and functional relevance by qRT-PCR. Consistent with the previous reports, upregulated SlbZIP1 was observed under cold stress $\left(4{ }^{\circ} \mathrm{C}\right)$, but downregulated expression was detected under heat stress $\left(40{ }^{\circ} \mathrm{C}\right)$ (Fig. 2a). The transcript of SlbZIP1 was also upregulated by $\mathrm{NaCl}$ (mainly in roots) and $\mathrm{H}_{2} \mathrm{O}_{2}$, while no significant change was detected when subjected to dehydration stress (Fig. 2a). Moreover, transcript levels of SlbZIP1 increased with $\mathrm{ABA}, \mathrm{ACC}, \mathrm{GA}$ and SA treatments, while IAA treatment could not induce its transcript (Fig. 2b). The

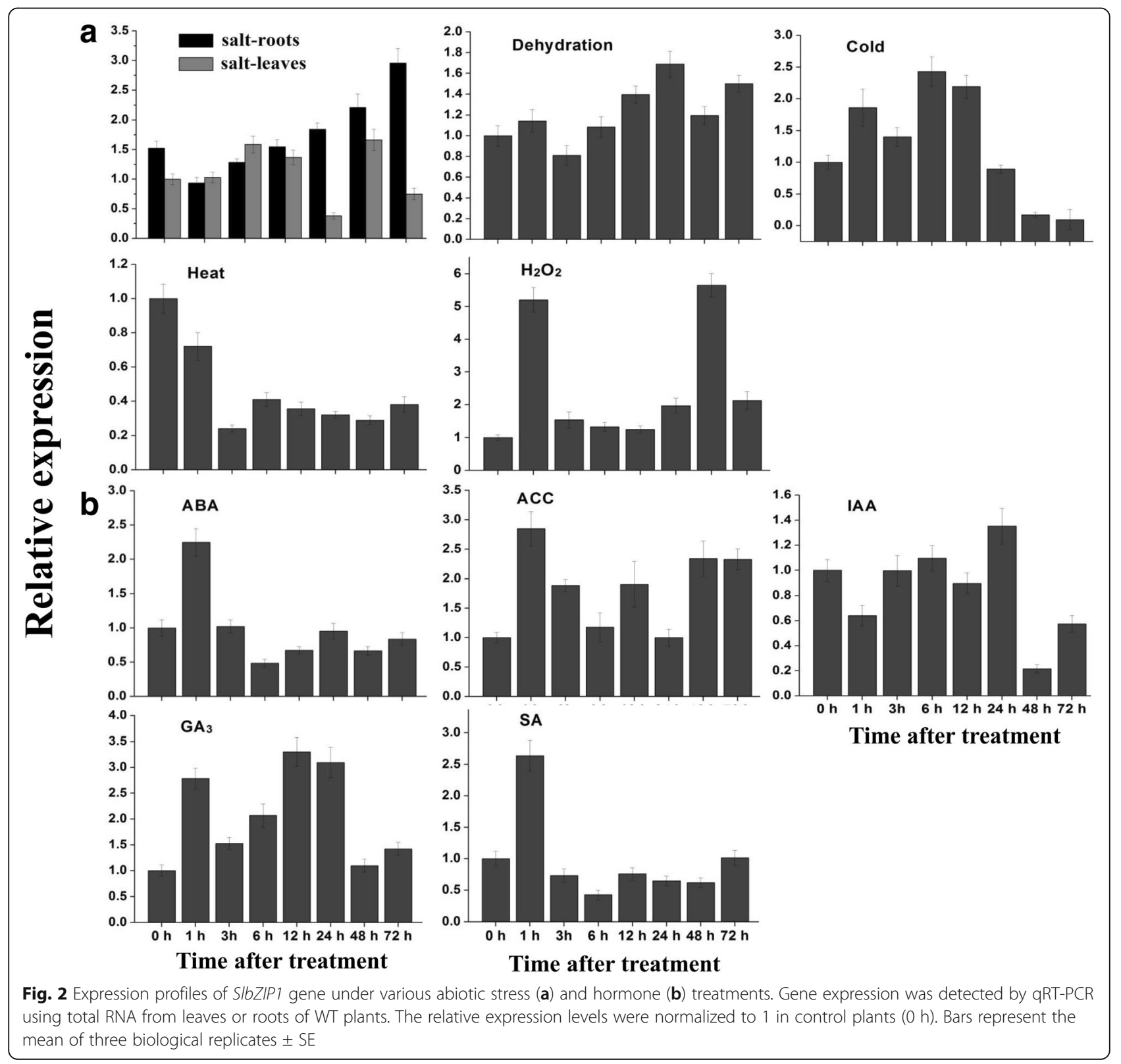


results indicate that SlbZIP1 makes possible regulation on gene expression related to hormone and stress response in tomato.

Silencing of SIbZIP1 downregulates the expression of ABA biosynthesis- and signal transduction-related genes

Since SlbZIP1 was remarkably induced by ABA and salt stress, to examine its functional roles in greater depth, 9 independent SlbZIP1-RNAi lines were produced. The accumulation of SIbZIP1 transcript was remarkably decreased to roughly $6-14 \%$ (Fig. 3a) of control levels in three RNAi lines (T3 generation, lines 2, 4 and 6), and thus they were chosen for further investigation. To verify specific repression of SlbZIP1, the expression levels of SlbZIP07 (NM_001247576), SlbZIP10 (AK327140) and SlbZIP39 (AK326780) were monitored because they are the most similar $(52.6,45.0$ and $69.3 \%$ similarity at the nucleotide level, respectively) tomato genes to SlbZIP1. The results showed that SIbZIP10 and SlbZIP39 mRNA levels were a little lower in transgenic plants than that of WT, while SlbZIPOT mRNA remained unchanged (Additional file 1: Figure S1). We further

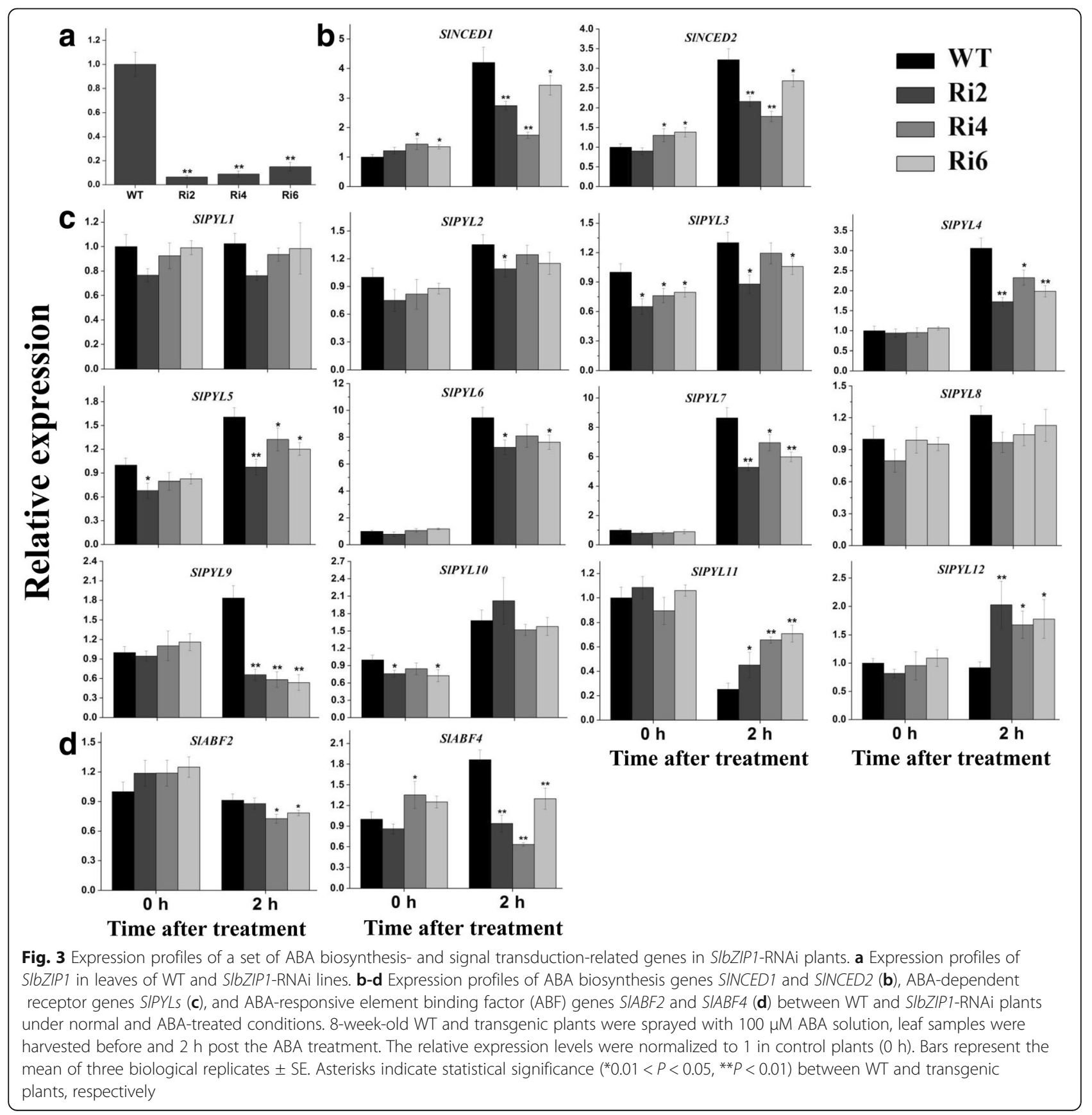


conducted a multiple sequence alignment among SlbZIP1, SlbZIP07, SlbZIP10 and SlbZIP39, the results indicate that the 435 bp DNA fragment of SlbZIP1 used in the hairpin is specific (Additional file 2: Figure S2). The results indicate that SlbZIP1 mRNA is specifically targeted by the SlbZIP1-RNAi transgene RNA.

The increased transcription of SlbZIP1 under ABA treatment impelled us to detect whether silencing of SlbZIP1 affects ABA biosynthesis and/or signal transduction in transgenic lines. The expression of SINCED1 and SINCED2 genes involved in ABA biosynthesis [28] was increased in transgenic plants grown under control conditions $(0 \mathrm{~h})$, while both of their expression were significantly downregulated in transgenic plants after $2 \mathrm{~h}$ of ABA treatment (Fig. 3b). Moreover, recent research conducted by ABA signaling shows that the primary ABA receptor is a central signaling module consisting of three classes of proteins, such as PYR/PYL/RCAR [29]. Then the transcription levels of 12 tomato PYL genes were detected between WT and transgenic plants. Wherein, no obvious difference in SIPYL1, SlPYL2 and SIPYL8 expression was detected for SlbZIP1-RNAi and WT plants under both conditions. On the contrary, the accumulation of SIPYL3 and SIPYL5 expression was reduced in different extents in transgenic plants under both conditions. Moreover, downregulated expression of SIPYL4, SIPYL6, SIPYL7 and SIPYL9, and upregulated expression of SIPYL11 and SIPYL12 in transgenic plants were detected at $2 \mathrm{~h}$ after ABA treatment, while reduced SIPYL10 expression was observed mainly under control conditions (Fig. 3c). Moreover, $\mathrm{ABF}$ (ABA-responsive element binding factors) genes are also the important downstream components of ABA signaling [30]. The transcription of two ABF genes, SlABF2 and SlABF4 was also markedly reduced in transgenic plants (Fig. 3d).

\section{Silencing of SIbZIP1 significantly decreases salt tolerance} Since tomato SlbZIP1 gene responded to various abiotic stresses, the effects of salt stress on WT and SlbZIP1RNAi tomato were investigated in soil. No abnormal morphological phenotype between WT and SlbZIP1-RNAi plants was observed under normal conditions $(0 \mathrm{~d}$, Fig. $4 \mathrm{a}$, upper and lower). However, transgenic plants exhibited more notorious $\mathrm{NaCl}$-induced symptoms under $400 \mathrm{mM}$ $\mathrm{NaCl}$, such as more wilting, especially in the lower leaves. The symptoms were more serious after $8 \mathrm{~d}$ of salt treatment with wilt of lower leaves in SlbZIP1-RNAi plants (Fig. 4b). Moreover, most of the leaves of transgenic plants displayed severe necrosis after $16 \mathrm{~d}$ exposed to salt stress, while the upper leaves of WT tomato showed less wilting at this time point (Fig. 4c). Moreover, after re-watering for 5 d, a lower survival rate of SlbZIP1-RNAi plants was observed (Fig. 4d).
To investigate the potential physiological mechanisms responsible for the reduced salt stress tolerance in SlbZIP1-RNAi plants, multiple stress-related physiological parameters were detected under normal and salt conditions. Changes were found in ABA, chlorophyll and malondialdehyde (MDA) contents, and CAT activity, which are typical parameters to evaluate plant stress tolerance. No obvious difference in these parameters was detected between WT and SlbZIP1-RNAi plants under normal conditions $(0 \mathrm{~d})$ except for proline levels (Figs. 4e-i). Upon exposure to salt stress, no distinct changes in $A B A$ contents were observed between WT and transgenic plants at $8 \mathrm{~d}$ post-treatment, while ABA contents in SlbZIP1RNAi plants were significantly lower than that of WT after $16 \mathrm{~d}$ of salt treatment (Fig. 4e). In addition, detectable reductions were also found in chlorophyll content (Fig. 4f) and CAT activity (Fig. 4g) in transgenic tomato leaves compared with WT plants. The MDA content was enhanced by salt stress, whereas the enhanced level in WT plants was lower than in transgenic lines (Fig. 4h). Furthermore, no significant change in proline contents was detected between WT and SlbZIP1-RNAi lines under salt stress (Fig. 4i). The data suggest that the expression of SlbZIP1 correlates with the degree of salt tolerance in transgenic tomato.

\section{Silencing of SIbZIP1 significantly reduces drought resistance}

To further test the influences of SlbZIP1 gene on drought resistance, a drought experiment was conducted by irrigated 20\% PEG6000. Before the PEG stress $(0 \mathrm{~d})$, WT and SlbZIP1-RNAi plants displayed similar growth status (Fig. 5a, upper and lower). The leaves of both WT and transgenic plants began to exhibit leaf wilting with chlorophyll loss after $10 \mathrm{~d}$ of dehydration stress, while transgenic plants displayed more severe symptoms compared with control plants (Fig. 5b). Apparent difference was detected on the 20th day after dehydration treatment, most leaves of transgenic plants showed serious wilting, whereas WT plants were less affected (Fig. 5c). Moreover, a lower survival rate of SlbZIP1-RNAi plants than that of WT plants was observed after watering for $5 \mathrm{~d}$ (Fig. $5 \mathrm{~d}$ ). We also measured chlorophyll contents between WT and transgenic lines during the drought assay and found that, the chlorophyll reduction in transgenic lines was higher than that in WT plants both at 10 and $20 \mathrm{~d}$ post-treatment (Fig. 5e). And lower CAT activity in transgenic leaves compared with WT leaves was detected following the drought assay (Fig. 5f). Furthermore, the increase of MDA content in WT plants was lower than in transgenic lines during the post-drought treatment (Fig. 5g). In addition, no significant change in proline levels was detected between WT and SlbZIP1-RNAi plants under the same 


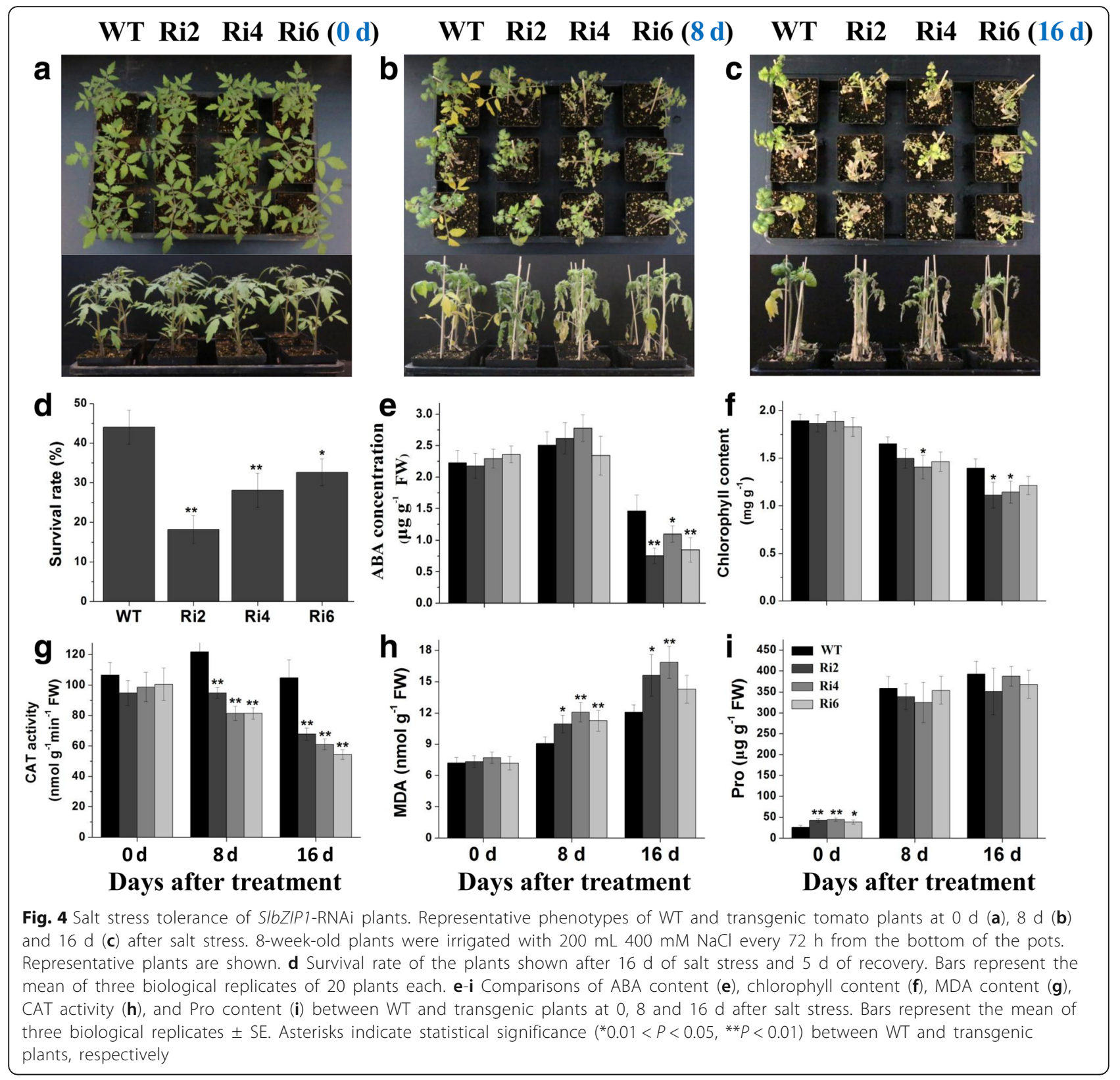

drought assay (Fig. 5h). The data indicate that SlbZIP1 functions importantly in tomato drought resistance.

Differentially expressed genes (DEGs) based on RNA-Seq data between WT and SIbZIP1-RNAi transgenic plants

To gain further insights into the potential mechanisms of the reduced salt and drought tolerance in SlbZIP1RNAi lines, the gene expression between WT and transgenic lines (Ri2) treated with salt stress for $1 \mathrm{~d}$ was determined and compared using RNA sequencing. A total of 395 genes were identified as DEGs (280 downregulated and 115 upregulated) that exhibited a > twofold difference in expression in transgenic lines
(Fig. 6, Additional file 3: Table S1), compared with the expression in WT plants. From the DEGs with function information, we found that over 50 genes are associated with biotic and abiotic stresses. The representative genes with reduced expression in leaf tissues are shown in Table 1. It is noteworthy that most of these genes encode proteins that respond to abiotic stress [e.g. MAP kinase kinase kinase (Solyc01g111880)], oxidative stress [e.g. peroxidase (Solyc08g069040)], biotic stimuli [e.g. pathogenesis-related proteins (e.g. Solyc02g031950 and Solyc04g007780), and protease inhibitor (e.g. Solyc03g020030 and Solyc03g020040)], and multiple TF genes [e.g. Trihelix TF (Solyc09g009250), Zinc finger protein TF (Solyc01g087050, Solyc05g025570 and 


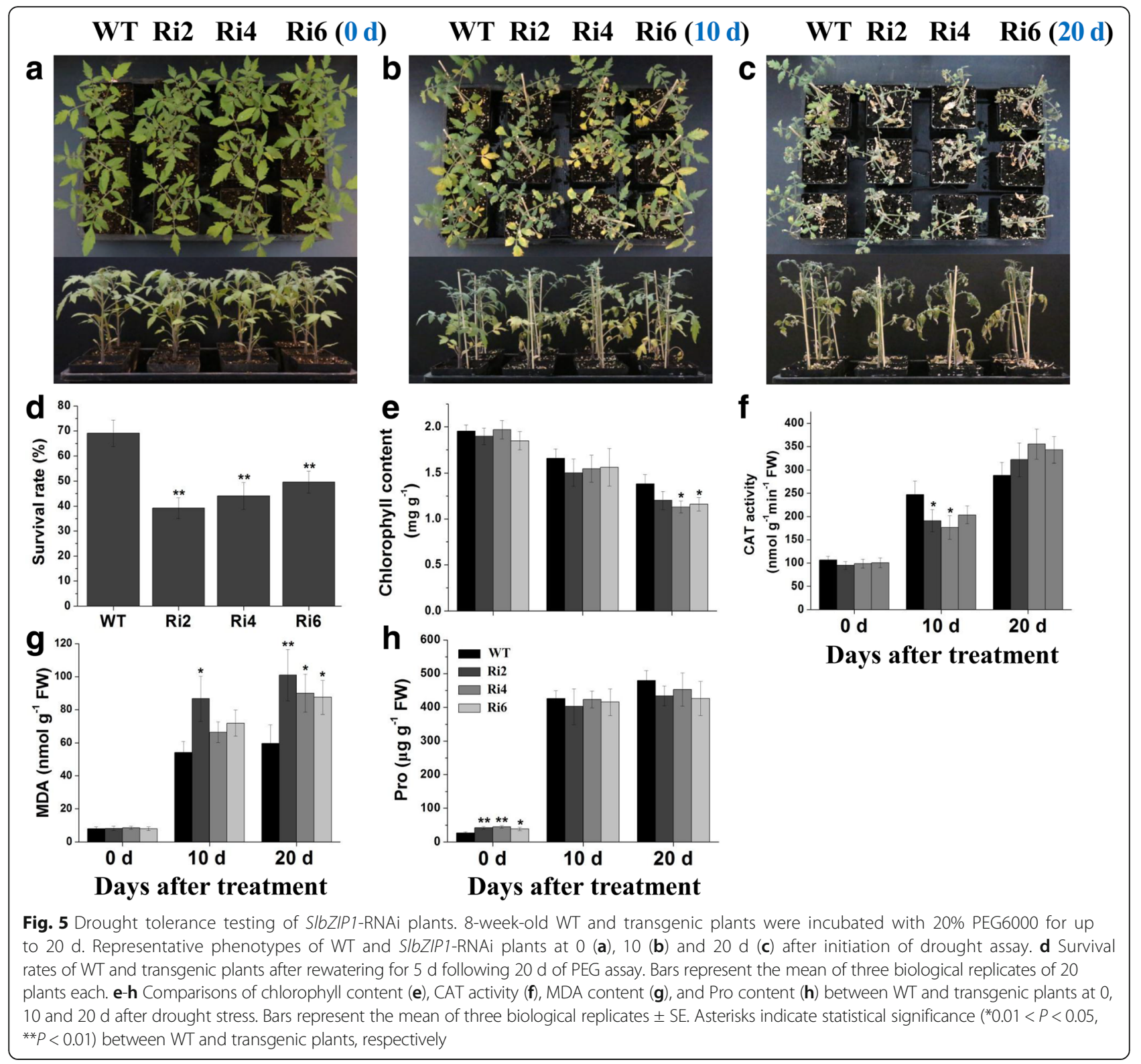

Solyc01g067360) and MYB TF (Solyc11g065840, Solyc11g011050 and Solyc01g010910)] were also detected.

Furthermore, 9 stress-related genes were selected and their expression was compared between WT and SlbZIP1-RNAi plants by qRT-PCR. With the exception of the elevated expression of SlMYB106 (Solyc01g010910) [31] in transgenic plants, the expression of most genes selected for confirmation displayed expected transcript accumulations in transgenic plants, despite the changes of several genes exhibiting some differences among the transgenic plants (Fig. 7), supporting the outcomes of the RNA-seq analysis and the thesis that tomato SlbZIP1 is involved in regulating stress-related genes. Wherein, the transcription levels of stress-related endochitinase gene (Solyc10g074440) [32], peroxidase gene (Solyc08g069040)
[33], potassium channel KAT3-like gene (Solyc01g010080) [34] and MAP kinase kinase kinase gene SIMAPKKK11 (Solyc01g111880) [35] were downregulated remarkably in transgenic lines. The expression of bZIP TF gene SlbZIP53 (Solyc10g050210) [20] was also reduced in transgenic plants. Nevertheless, no obvious difference in the expression of MYB TF gene SlMYB46 (Solyc11g065840) [31] and Trihelix TF gene SlGT26 (Solyc09g009250) [36] was observed. Furthermore, the transcription of a pathogenesisrelated protein gene SISTH-2 (Solyc02g031950) [37] in SlbZIP1-RNAi plants was also downregulated (Fig. 7). These data indicate that silencing of SlbZIP1 exerts influence on the transcription levels of a series of stress-related genes and hence results in reduced salt and drought tolerance in SlbZIP1-RNAi tomato. 


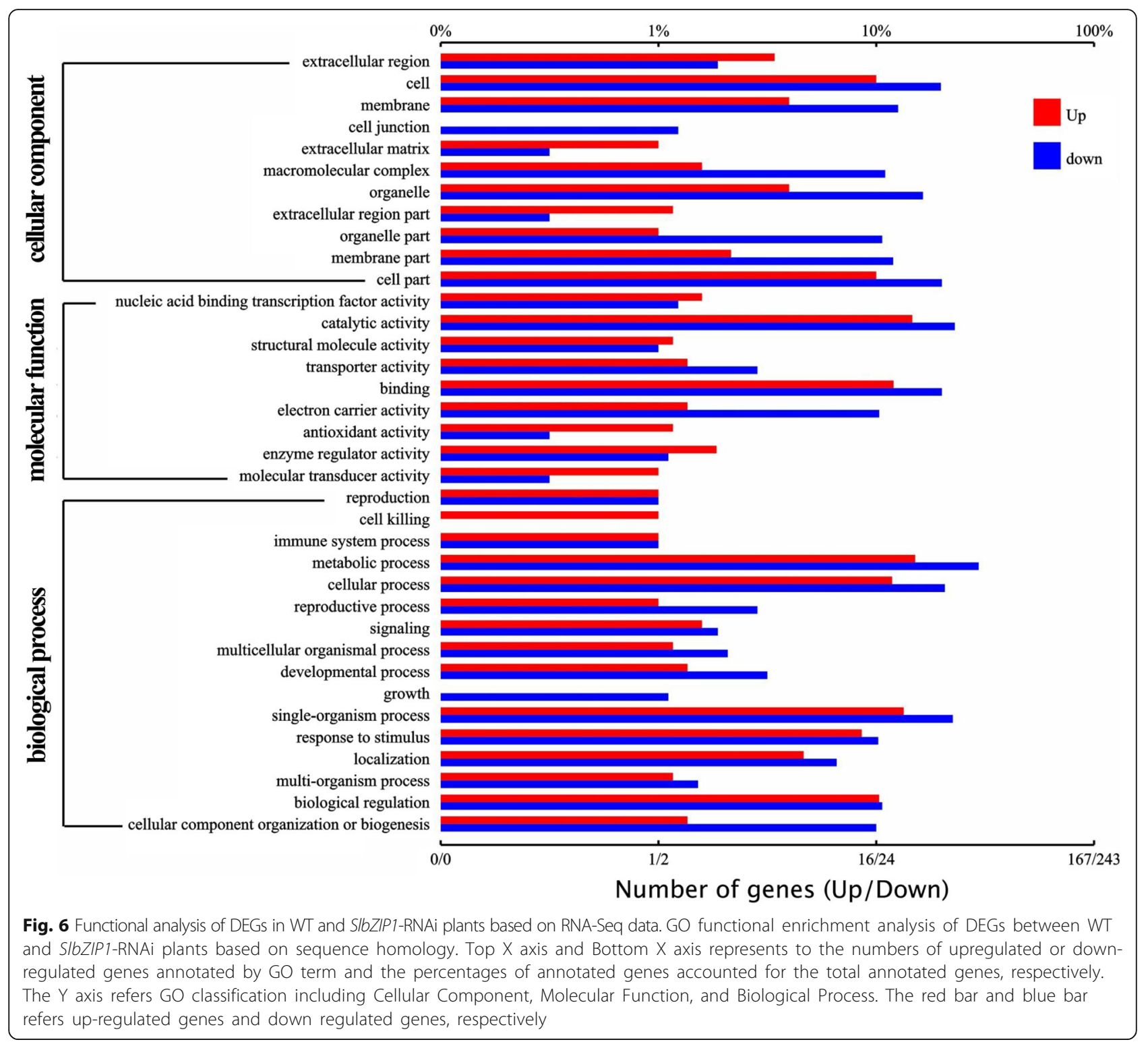

\section{Discussion}

The specific mechanisms of the ABA signaling network and stress responses in tomato, compared with the model plants Arabidopsis and rice remain largely unknown. bZIP TFs undoubtedly represent one of the most significant participants in the regulation of stress tolerance, which act pivotal roles in diverse physiological processes, including stress responses and post-stress recovery [3]. Though the participation of several tomato bZIP genes such as SIAREB1, SIAREB2 [21, 22] and LebZIP2 [26] in abiotic stress response has been reported, the biological roles of most tomato bZIPs remain unclear. Previously, expression analysis suggests that SlbZIP1 transcript is induced by wounding, and ABA may act to curtail its wounding-induced synthesis $[20,27]$, whereas its roles in abiotic stress response remain undefined. In this study, we further confirmed that SlbZIP1 expression was significantly improved by $\mathrm{NaCl}$, cold and ABA (Fig. 2), suggesting that SlbZIP1 may respond to various environmental cues. Furthermore, we presented the evidence that expression of Group A bZIP gene SlbZIP1 correlated with the degrees of salt and drought tolerance testified by SlbZIP1-RNAi transgenic plants (Figs. 4 and 5). Furthermore, we determined that SlbZIP1 was involved in regulating a variety of biotic and abiotic stress-related genes (Fig. 7, Additional file 3: Table S1). Recent works showed that group A of bZIPs, including Arabidopsis ABF1-4 and ABI5, and rice TRAB1, OsbZIP23, OsABF1 and OsABI5, participates in ABA-mediated and stress-related gene transcription [18], indicating that they may be involved in regulating ABA-dependent stress response pathways. Therefore, the conservation of group $\mathrm{A}$ 
Table 1 RNA sequencing analysis of selected genes downregulated $(P \leq 0.05)$ in SIbZIP1-RNAi transgenic line Ri2 compared with WT tomato plants

\begin{tabular}{|c|c|c|c|}
\hline Accession & Gene product & GO term & Log2 fold (Ri2/WT) \\
\hline Solyc07g007450 & Iron-sulfur cluster assembly protein & iron ion binding & -7.821905209 \\
\hline Solyc10g080580 & Receptor-like kinase & protein phosphorylation & -7.371740136 \\
\hline Solyc02g067770 & Cysteine-rich receptor-like protein kinase 2 & protein phosphorylation & -7.212299904 \\
\hline Solyc02g082990 & Stress-induced protein KIN2 & Stress response & -7.12544498 \\
\hline Solyc11g064780 & F-box/LRR-repeat protein & Disease resistance & -7.033024239 \\
\hline Solyc07g005040 & $\mathrm{H}^{+}$-ATPase & ATP binding & -6.828267133 \\
\hline Solyc09g009250 & Trihelix transcription factor PTL & Regulation of transcription & -6.828267133 \\
\hline Solyc02g086960 & Receptor-like kinase & protein phosphorylation & -4.80337823 \\
\hline Solyc01g087050 & Zinc finger protein WIP2-like & Regulation of transcription & -4.746313437 \\
\hline Solyc02g093540 & Cytochrome P450 & Controlling growth. Biotic stress & -4.369713338 \\
\hline Solyc01g010080 & Potassium channel KAT3-like & potassium channel activity & -4.296148364 \\
\hline Solyc12g009190 & Receptor-like protein kinase 3 & protein phosphorylation & -4.136708132 \\
\hline Solyc11g065840 & MYB transcription factor & Regulation of transcription & -3.719575489 \\
\hline Solyc05g047680 & Cytochrome P450 & Controlling growth. Biotic stress & -3.591453231 \\
\hline Solyc10g074440 & Endochitinase & Stress response & -3.131124702 \\
\hline Solyc11g011050 & MYB transcription factor & Regulation of transcription & -2.932906604 \\
\hline Solyc02g085130 & Tubby-like F-box protein & Disease resistance & -2.871978777 \\
\hline Solyc09g084460 & Wound-induced proteinase inhibitor 1 & Stress response & -2.844760217 \\
\hline Solyc02g031950 & Pathogenesis-related protein & Response to biotic stimulus & -2.815681677 \\
\hline Solyc05g025570 & Zinc finger protein & Regulation of transcription & -2.69118912 \\
\hline Solyc08g069040 & Peroxidase 1 & Response to oxidative stress & -2.480134415 \\
\hline Solyc01g097630 & F-box protein & Disease resistance & -2.411524305 \\
\hline Solyc08g013760 & Putative F-box protein & Disease resistance & -2.33246778 \\
\hline Solyc01g010910 & MYB transcription factor & Regulation of transcription & -2.319000953 \\
\hline Solyc00g011160 & Cytochrome P450 like & Controlling growth. Biotic stress & -2.256203387 \\
\hline Solyc01g111880 & MAP kinase kinase kinase 11 & Biotic and abiotic stress response & -2.14925363 \\
\hline Solyc03g020030 & Proteinase inhibitor II & Stress response & -2.146031647 \\
\hline Solyc01g066910 & Lipid-transfer protein DIR1 & Response to stress & -1.958613976 \\
\hline Solyc04g007780 & Pathogenesis-related protein & Response to biotic stimulus & -1.762833313 \\
\hline Solyc10g050210 & bZIP transcription factor & Regulation of transcription & -1.762190276 \\
\hline Solyc03g020040 & Proteinase inhibitor II & Stress response & -1.707542181 \\
\hline Solyc01g067360 & Zinc finger protein & Regulation of transcription & -1.701869583 \\
\hline Solyc03g034390 & Lipid-transfer protein & Response to stress & -1.684560398 \\
\hline
\end{tabular}

bZIPs regulatory systems for ABA-dependent stress responses has been proposed.

The surveys that SlbZIP1-RNAi plants displayed reduced salt and drought tolerance as presented by lower survival ratio, worse growth performance and significantly reduced transcription of many stress-associated genes under salt and drought stresses, were presented. The results demonstrated that tomato SlbZIP1 functions as a positive regulator of salt and drought stress tolerance. Similarly, previously tomato SIAREB1 was reported to play an essential role in improving salt and drought tolerance demonstrated by overexpression and silencing in transgenic tomato [22]. Besides, SlbZIP1 protein shares a close homolog with Arabidopsis bZIP53, the heterodimers of Arabidopsis bZIP53 and bZIP1 reprogram amino acid metabolism under low energy stress [38]. The possible mechanisms accounting for the changed stress tolerance in SlbZIP1-RNAi lines could be illustrated in part by multiple physiological parameters detected in this study. In general, as a crucial stress hormone, high degrees of ABA may accelerate and/or strengthen stress responses, which are associated with 


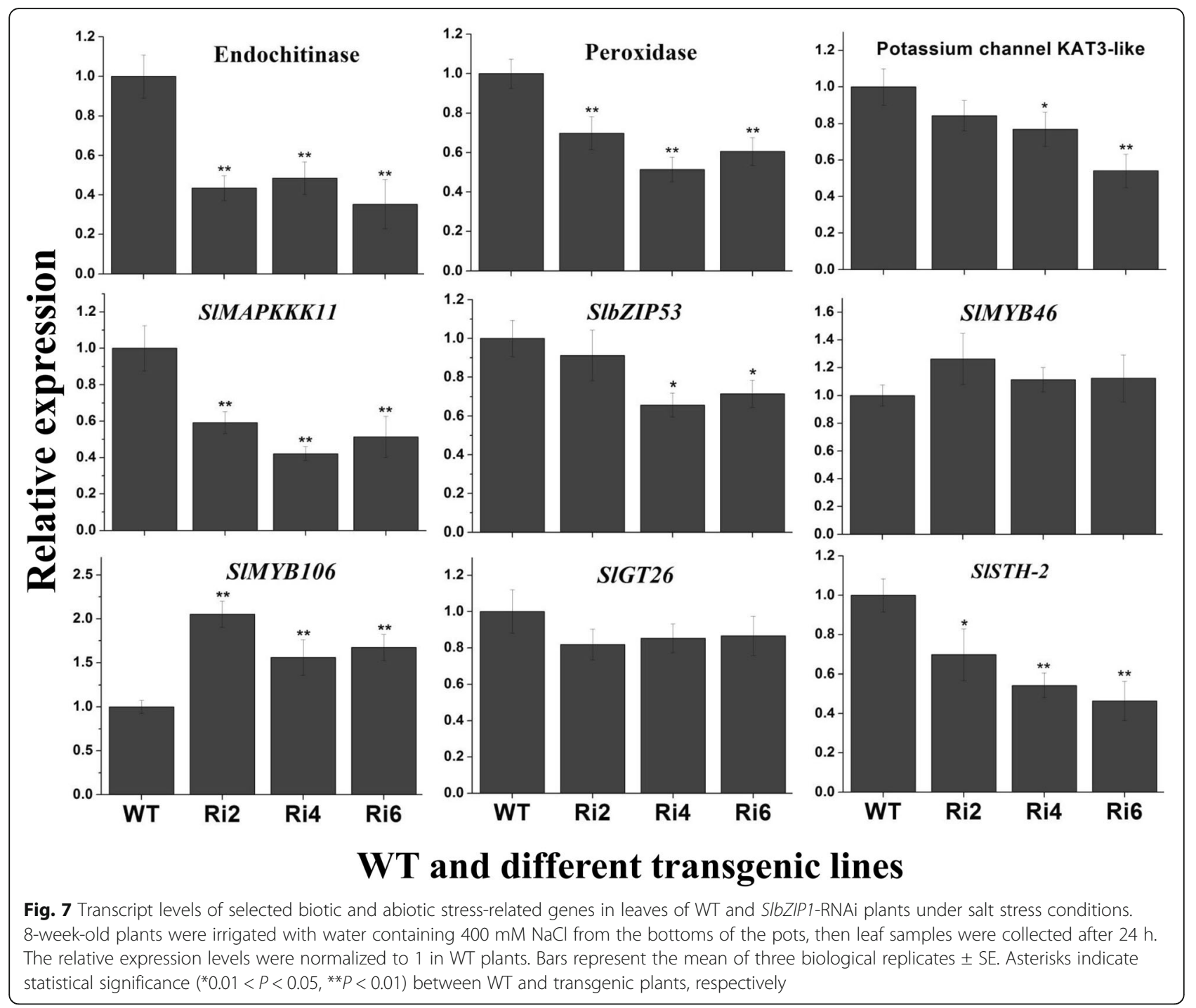

enhanced stress tolerance [1, 39]. Transgenic lines tended to accumulate less ABA than that of WT plants under salt stress (Fig. 4), which partly explained the reduced salt tolerance of transgenic plants. Proline acts as the regulator of antioxidant system or molecular chaperone to stabilize proteins [40], while it was not obviously changed in transgenic plants under both stress conditions (Figs. 4 and 5). Moreover, chlorophyll content is regarded as an indicator of the stress damage done to the photosynthetic apparatus. And stress conditions including salt and drought often generate ROS (reactive oxygen species) in plants, hence inducing damages to membrane lipids, proteins and nucleic acids. Therefore, enzymatic defending systems are adapted by plants for detoxifying ROS synthesized. Wherein, CAT is involved in the main defense system against $\mathrm{H}_{2} \mathrm{O}_{2}$ accumulation and toxicity [41-43]. This work exhibited that both chlorophyll content and CAT activity in SlbZIP1-RNAi lines were lower than that in WT under both stresses (Figs. 4 and 5). The reduced activity of antioxidant enzyme might underlie the higher $\mathrm{H}_{2} \mathrm{O}_{2}$ contents in SlbZIP1-RNAi plants. Furthermore, cell membranes are one of the primary targets of various environmental cues, and MDA is usually employed as an index of oxidative damages in plant $[44,45]$. The present survey showed that MDA contents in transgenic plants were higher than that in WT tomato under both stresses (Figs. 4 and 5). In this context, increased MDA levels have also been detected in SIAREB1-antisense tomato plants [22]. These data suggest that SlbZIP1-RNAi plants have less robust photosynthetic abilities and more oxidative damage than WT plants, thus exhibiting reduced tolerance to salt and drought stresses.

Numerous molecular and biochemical studies have shown that ABA accumulates in various plant tissues to adapt to drought and salinity stresses, and is believed to 
act as an essential regulator in abiotic stress response $[39,46]$. The results indicated that SlbZIP1 might act as a positive regulator in stress response by an ABAmediated pathway. Firstly, treatment of exogenous ABA significantly affected the expression of SlbZIP1 (Fig. 2), similar to previously reported ABA-responsive tomato LebZIP2 gene, and its overexpression improves NOA1 and $N R$ transcription in the $N$. benthamiana leaves [26]. In this context, downregulated transcriptions of ABA biosynthesis-related genes including SINCED1 and SINCED2, which are regarded as the rate-limiting step in the stress-induced ABA synthesis pathway [30, 47] were detected in SlbZIP1-RNAi plants (Fig. 3). The result is similar to the data that the overexpression of grape VlbZIP36 gene obviously improves AtNCED3 expression in transgenic Arabidopsis [48]. The endogenous ABA content of SlbZIP1-RNAi lines was also remarkably lower than that of WT exposed to salt treatment (Fig. 4e). Furthermore, the expression of most of detected tomato PYL genes encoding ABA-dependent receptors and two ABF genes SlABF2 and SlABF4 were also downregulated in SlbZIP1-RNAi plants (Fig. 3). The data indicate that silencing of SlbZIP1 may affect ABA biosynthesis/signal transduction through regulating directly or indirectly the relevant gene expression.

The transcriptions of a series of biotic- and abiotic stress-related genes were differentially expressed in SlbZIP1-RNAi lines (Additional file 3: Table S1, Fig. 7). The transcription of stress-related genes leads to alterations of biochemical and physiological pathways that are pivotal for plants adaptation to unfavorable conditions. In this study, remarkably downregulated expression of multiple stress-relevant genes, including endochitinase gene [32], peroxidase gene [33], potassium channel KAT3-like gene [34] and SlMAPKKK11 gene [35] were detected in transgenic lines. The stress-related TF gene SlbZIP53 was also reduced in transgenic plants, and it has been well documented that bZIP TFs participate in diverse biotic and abiotic stress responses in multiple plant species [20]. Interestingly, the transcription levels of a pathogenesisrelevant gene SlSTH-2 [37], were also reduced in SlbZIP1-RNAi plants. Thus, the results indicate that SlbZIP1, besides playing a role in tomato adaptation to abiotic stresses, may also integrate signals derived from both biotic and abiotic stresses, while further clarification is required. Thus, the decreased tolerance to salt and drought assays detected in the present study can be, at least partially, associated with SlbZIP1-regulated downstream genes. The results are in line with previous studies, in which improved tolerance to stress is attributed to upregulated expression of multiple biotic and abiotic stress-relevant genes in the LebZIP2- and SlAREB1-overexpressing lines [5, 22, 26]. In addition, since ABA contents in plants are improved in response to abiotic stress such as salt and drought, resulting in transcription of stress-related genes, thus the reduced $A B A$ accumulation in SlbZIP1-RNAi plants will restrict ABAdependent pathways that regulate the expression of many stress-related genes $[39,49]$. Collectively, we suppose that SlbZIP1 mediates signaling pathways to cope with abiotic stress through modulating the transcription of stress-responsive genes, thus silencing of SlbZIP1 resulting in a reduced salt and drought tolerance in SlbZIP1-RNAi lines. Combining these results, we propose a hypothetical molecular mechanism for SlbZIP1 functions in salt and drought stress tolerance in tomato, as the scheme representing in Additional file 4: Figure S3.

\section{Conclusions}

Collectively, the data presented in this study not only reveal an important regulatory function for tomato SlbZIP1 in salt and drought stress tolerance, but also provide a foundation for further investigation of salt- and drought-induced signaling pathways in which SlbZIP1 participates. Thus SlbZIP1 may hold a potential application in the engineering of salt- and drought-tolerant cultivars. However, functional investigation of SlbZIP1-overexpressioning transgenic plants is necessary to further determine the functions of tomato SlbZIP1 in stress tolerance. Moreover, further explorations on the identification of SlbZIP1-regulated downstream genes will be useful to clarify the mechanisms of SlbZIP1 in the regulation of salt and drought stress tolerance.

\section{Methods}

\section{Plant materials and growth conditions}

The WT tomato (Solanum lycopersicom Mill. cv. Ailsa Craig) and transgenic seeds (T3 generation) were sterilized before germinating and the seedlings were then sown in commercially sterilized soil mix (peat moss, perlite, and vermiculite, 3:1:1, v/v/v). The plants were cultivated in a greenhouse under sodium lights timed for $16 \mathrm{~h}$ days $\left(27{ }^{\circ} \mathrm{C}\right)$ and $8 \mathrm{~h}$ nights $\left(18{ }^{\circ} \mathrm{C}\right)$. The tissues including roots, stems, flowers, and leaves and fruits of individual period were gathered as described before [50]. All the plant samples were collected and immediately frozen in liquid nitrogen and stored at $-80{ }^{\circ} \mathrm{C}$.

\section{Plant abiotic stress and hormone treatments}

Tomato seedlings were grown in a greenhouse under the same conditions mentioned above, and all the treatment experiments were conducted using potted 35 day-old plants chose according to their uniformity. Plants were irrigated with a $1 \mathrm{~g} \mathrm{~L}^{-1}$ Murashige and 
Skoog nutrient solution once per week. Untreated plants were used as controls, and leaves and roots were collected at $1,3,6,12,24,48,72 \mathrm{~h}$ after each treatment. In each case, individual plants were used for each timepoint and treatment in triplicate and a total of 21 plants were employed in each treatment. Salt stress was conducted by submerging the roots of plants in $200 \mathrm{mM} \mathrm{NaCl}$, then leaves and roots were harvested. For dehydration essay, plants were gently pulled out and washed carefully with water to remove soil, and left on a piece of dry filter paper at $25 \pm 1^{\circ}$ $\mathrm{C}$, and then the leaf samples were collected. "Cold" and "heat" stress essays were applied by incubating the plants at 4 and $40{ }^{\circ} \mathrm{C}$, respectively [51]. For oxidative stress, the plants were sprayed with $10 \mathrm{mM}$ $\mathrm{H}_{2} \mathrm{O}_{2}$ solution. For hormone treatments, the plants were sprayed with $0.1 \mathrm{mM} \mathrm{ABA}, \mathrm{ACC}, \mathrm{GA}_{3}$, IAA, and $2 \mathrm{mM}$ SA solution and left for $1,3,6,12,24,48$, $72 \mathrm{~h}$ at $25 \pm 1{ }^{\circ} \mathrm{C}$, and then the leaves were collected. All the collected samples were frozen immediately in liquid nitrogen and stored at $-80{ }^{\circ} \mathrm{C}$.

\section{RNA extraction and qRT-PCR analysis}

Total RNA was extracted by the Trizol reagent (Invitrogen, Shanghai, China). $2 \mu \mathrm{g}$ RNA was reversetranscribed using PrimeScript reverse transcriptase (with gDNA Eraser for Perfect Real Time) (TaKaRa, Dalian, China) with the mix of Oligo dT Primer and Random 6 mers. The qRT-PCR reaction consisted of $3.2 \mu \mathrm{L}$ distilled water, $5 \mu \mathrm{L} 2 \times$ SYBR Premix Ex Taq II (Tli RNaseH Plus) (TaKaRa, Dalian, China), $0.4 \mu \mathrm{L}$ $10 \mathrm{mM}$ each primer and $1 \mu \mathrm{L}$ cDNA. qRT-PCR was conducted using the CFX96 ${ }^{\text {th }}$ Real-Time System (BioRad, USA) under the procedures below: $95{ }^{\circ} \mathrm{C} 30 \mathrm{~s}$, 40 cycles of $95{ }^{\circ} \mathrm{C} 5 \mathrm{~s}, 60{ }^{\circ} \mathrm{C} 1 \mathrm{~min}$, followed by a melting curve analysis. Tomato $C A C$ gene and EF1 $\alpha$ gene were selected as the internal standard for organ expression and abiotic stress analysis, respectively $[52,53]$.

\section{Construction of RNAi vector and tomato transformation}

The SlbZIP1-RNAi vector was constructed using the pBIN19 driven by the CaMV $35 \mathrm{~S}$ promoter (Additional file 5: Figure S4). The 435 bp SlbZIP1-specific fragment was amplified using the primers SlbZIP1RNAi-F/R (Additional file 6: Table S2), and confirmed by sequencing. The obtained construct was transformed into WT tomato (Ailsa Craig) by Agrobacterium tumefaciens (LBA4404)-mediated transformation of cotyledon explants, and transformed plants were selected for kanamycin resistance $\left(50 \mathrm{mg} \mathrm{L}^{-1}\right)$. DNA was isolated based on the method of Genomic DNA Extraction Kit (Invitrogen, Shanghai, China), and then analyzed by PCR to ascertain the presence of T-DNA using the primers NPTII-F/R (Additional file 6: Table S2).

\section{Phenotype analyses for salt and drought stress tolerance} and $A B A$ treatment assays

WT and SlbZIP1-RNAi plants were pre-grown in seedling nurseries for $14 \mathrm{~d}$ and transferred to pots in the greenhouses under the conditions described above. Then 8week-old plants of similar size were selected for salt and drought tolerance assays. The plants were irrigated from the bottom of the pots with $400 \mathrm{mM} \mathrm{NaCl}(200 \mathrm{~mL})$ or $20 \%$ PEG6000 (200 mL) every $72 \mathrm{~h}$. Survival rate was calculated after $5 \mathrm{~d}$ of recovery from both stresses. Plants were considered as survivals if there were healthy and green young leaves after $5 \mathrm{~d}$ of rewatering. For the ABA treatment of the transgenic plants, the plants of similar size were sprayed with $100 \mu \mathrm{M}$ ABA solution and left for $2 \mathrm{~h}$ at $25 \pm$ $1{ }^{\circ} \mathrm{C}$, while untreated plants were used as controls.

\section{Evaluation of salt stress tolerance}

ABA was quantified using a high-performance liquid chromatography (HPLC, Rigol L3000), ABA measurements were conducted as described before [54]. The leaf samples of soil-grown WT and SlbZIP1-RNAi plants under normal and salt treatment for $8 \mathrm{~d}$ and $16 \mathrm{~d}$ were used for ABA measurement. Thermo solution 220 spectrophotometer was used for physiological parameter measurements at $25{ }^{\circ} \mathrm{C}$. The protocols for determination of chlorophyll (Chl) and malondialdehyde (MDA) content were as published previously [55]. Detection of CAT activities was assayed based on L Góth [56]. The reactions were initiated by adding $\mathrm{H}_{2} \mathrm{O}_{2}$ solution, and the activities were monitored by detecting the reduction in the absorbance of $\mathrm{H}_{2} \mathrm{O}_{2}$ at $240 \mathrm{~nm}$. Free proline contents in tomato leaves were detected as previously described [57].

\section{RNA sequencing and data analysis}

Eight-week-old WT and SlbZIP1-RNAi plants (mixed samples of six plants) were irrigated with water containing $400 \mathrm{mM} \mathrm{NaCl}$ from the bottoms of the pots, and then leaf samples were collected after $24 \mathrm{~h}$. Then RNAs extracted from the salt-treated leaves were employed for the RNA sequencing using Illumina HiSeq 2500 platform, and the 150 bp paired-end reads were generated by Genepioneer Bioinformatics Institute (Nanjing, China). The clean reads were filtered from the raw reads by removing duplication sequences, adaptor sequences and low-quality reads. Cleaned reads were subsequently mapped to the tomato reference genome by HISAT2 software [58]. The quantitative information of the RNA-seq data can be seen in Additional file 7: Table S3. Gene expression abundance was analyzed using StringTie software [59], to identify DEGs (differentially expressed genes), transcript abundance was normalized by FPKM (expected number of Fragments Per Kilobase of transcript sequence per Millions base pairs sequenced) method, and FDR (false discovery rate) $\leq 0.05$ was 
used to determine the threshold for DEGs. Then qRT-PCR was carried out to validate the results of RNA-seq, and gene-specific primers used for qRTPCR of selected stress-related genes were listed in Additional file 6: Table S2.

\section{Statistical analysis}

When appropriate, the results were analyzed by one-way analysis of variance (ANOVA) and means different were significant by a Dunnett's test at $P<0.05$ and $P<0.01$. Statistical analyses were conducted with SPSS software 20 version (IBM Corp., USA). In addition, we used a cut-off value of threefold for tissue-specific transcription and twofold for stress induction or repression [60].

\section{Additional files}

Additional file 1: Figure S1. Relative expression profiles of SIbZIP07, SIbZIP10 and SIbZIP39 in the leaves of WT and SIbZIP1-RNAi lines under normal conditions. (DOCX $77 \mathrm{~kb}$ )

Additional file 2: Figure S2. Multiple sequence alignment among SIbZIP1, SIbZIP07, SIbZIP10 and SIbZIP39 genes. (DOCX $570 \mathrm{kb)}$

Additional file 3: Table S1. Gene products of annotated genes or with sequence similarity showing at least 2 -fold change in transcript abundance $(p<0.05)$ in leaves of SIbZIP1-RNAi line Ri2 compared with WT plants. (XLSX $76 \mathrm{~kb}$ )

Additional file 4: Figure S3. Proposed model depicting the functions of SIbZIP1 in the regulation of salt and drought stress tolerance. (DOCX $102 \mathrm{~kb})$

Additional file 5: Figure S4. Hairpin construct of the SIbZIP1 gene for double-stranded RNAi vector. (DOCX $96 \mathrm{~kb}$ )

Additional file 6: Table S2. Specific primer sequences used for cloning procedure and qRT-PCR analysis. (DOCX $18 \mathrm{~kb}$ )

Additional file 7: Table S3. The quantitative information of the RNAseq data. (DOCX $17 \mathrm{~kb}$ )

\section{Abbreviations}

ABA: Abscisic acid; ABF: ABRE binding factor; ABRE: ABA responsive element; ACC: 1-aminocyclopropane-1-carboxylate; bZIP: Basic leucine zipper; CAT: Catalase; GA: Gibberellin acid; IAA: Indole-3-acetic acid; MDA: Malondialdehyde; Nr: Never ripe; Pro: Proline; qRT-PCR: quantitative reverse transcription-PCR; rin: Ripening inhibitor; ROS: Reactive oxygen species; SA: Salicylic acid; TF: Transcription factor; WT: Wild-type

\section{Acknowledgements}

All authors thank Prof G. P. Chen of Chongqing University for providing the $\mathrm{AC}^{++}$(Ailsa Craig) seeds. All authors are also grateful to the laboratory members for help, advice and discussion.

\section{Funding}

The funders had no role in the experiment design, data analysis, decision to publish or preparation of the manuscript. This work was supported by National Natural Science Foundation of China (no. 31700226; 31501352), Priority Academic Program Development of Jiangsu Higher Education Institutions (PAPD), Natural Science Foundation of Jiangsu Province (BK20160215), Natural science fund for colleges and universities in Jiangsu Province (16KJB210004) and Natural science fund of Jiangsu normal university (15XLR030).

\section{Availability of data and materials}

The raw data of RNA-seq experiments generated in this study have been deposited in the NCBI database under the accession number SRP119943.

\section{Authors' contributions}

$\mathrm{MZ}$ and $\mathrm{ZL}$ designed the experiments, $\mathrm{MZ}$ and $\mathrm{XM}$ analyzed the data and wrote the manuscript. XM, JC and GL performed the experiments, JC and TD repeated some of the experiments and revised the manuscript. All authors have read and approved the final manuscript.

Ethics approval and consent to participate Not applicable.

\section{Competing interests}

The authors declare that they have no competing interests.

\section{Publisher's Note}

Springer Nature remains neutral with regard to jurisdictional claims in published maps and institutional affiliations.

\section{Author details}

${ }^{1}$ School of Life Sciences, Jiangsu Normal University, 101 Shanghai Road, Xuzhou, Jiangsu Province 221116, People's Republic of China. ${ }^{2}$ Jiangsu Key laboratory of Phylogenomics \& Comparative Genomics, Jiangsu Normal University, Xuzhou, Jiangsu Province, People's Republic of China.

Received: 20 November 2017 Accepted: 26 April 2018

Published online: 08 May 2018

\section{References}

1. Zhu J-K. Abiotic stress signaling and responses in plants. Cell. 2016;167(2):313-24.

2. Shinozaki K, Yamaguchi-Shinozaki K. Gene networks involved in drought stress response and tolerance. J Exp Bot. 2007;58(2):221-7.

3. Banerjee A, Roychoudhury A. Abscisic-acid-dependent basic leucine zipper (bZIP) transcription factors in plant abiotic stress. Protoplasma. 2017;254(1):3-16.

4. Chen WJ, Zhu T. Networks of transcription factors with roles in environmental stress response. Trends Plant Sci. 2004;9(12):591-6.

5. Yánez M, Cáceres S, Orellana S, Bastías A, Verdugo I, Ruiz-Lara S, Casaretto JA. An abiotic stress-responsive bZIP transcription factor from wild and cultivated tomatoes regulates stress-related genes. Plant Cell Rep. 2009;28(10):1497-507.

6. Selvaraj D, Loganathan A, Ramalingam S. Molecular characterization and phylogenetic analysis of bZIP protein in plants. J Prot Bioinform. 2010;3:230-3.

7. Izawa T, Foster R, Chua N-H. Plant bZIP protein DNA binding specificity. J Mol Biol. 1993;230(4):1131-44.

8. Foster R, Izawa T, Chua N. Plant bZIP proteins gather at ACGT elements. FASEB J. 1994;8(2):192-200.

9. Zhang L, Zhang L, Xia C, Zhao G, Liu J, Jia J, Kong X. A novel wheat bZIP transcription factor, TabZIP60, confers multiple abiotic stress tolerances in transgenic Arabidopsis. Physiol Plant. 2015;153(4):538-54.

10. Jakoby M, Weisshaar B, Dröge-Laser W, Vicente-Carbajosa J, Tiedemann J, Kroj T, Parcy F. bZIP transcription factors in Arabidopsis. Trends Plant Sci. 2002;7(3):106-11.

11. Yoshida T, Fujita Y, Sayama H, Kidokoro S, Maruyama K, Mizoi J, Shinozaki K, Yamaguchishinozaki K. AREB1, AREB2, and ABF3 are master transcription factors that cooperatively regulate $A B R E-d e p e n d e n t ~ A B A$ signaling involved in drought stress tolerance and require ABA for full activation. Plant J. 2010;61 (4):672-85.

12. Zhong L, Chen D, Min D, Li W, Xu Z, Zhou Y, Li L, Chen M, Ma Y. AtTGA4, a bZIP transcription factor, confers drought resistance by enhancing nitrate transport and assimilation in Arabidopsis thaliana. Biochem Biophys Res Commun. 2015;457(3):433-9.

13. Yang O, Popova OV, Süthoff U, Lüking I, Dietz KJ, Golldack D. The Arabidopsis basic leucine zipper transcription factor AtbZIP24 regulates complex transcriptional networks involved in abiotic stress resistance. Gene. 2009;436(1-2):45-55

14. Lu G, Gao C, Zheng X, Han B. Identification of OsbZIP72 as a positive regulator of ABA response and drought tolerance in rice. Planta. 2009;229(3):605-15.

15. Xiang Y, Tang N, Du H, Ye H, Xiong L. Characterization of OsbZIP23 as a key player of the basic leucine zipper transcription factor family for conferring abscisic acid sensitivity and salinity and drought tolerance in rice. Plant Physiol. 2008;148(4):1938-52.

16. Liu C, Mao B, Ou S, Wang W, Liu L, Wu Y, Chu C, Wang X. OsbZIP71, a bZIP transcription factor, confers salinity and drought tolerance in rice. Plant Mol Biol. 2014;84(1):19-36. 
17. Xu D, Li J, Gangappa SN, Hettiarachchi C, Lin F, Andersson MX, Jiang Y, Deng XW, Holm M. Convergence of light and ABA signaling on the ABI5 promoter. PLoS Genet. 2014;10(2):e1004197.

18. Liang C, Meng Z, Meng Z, Malik W, Rong Y, Lwin KM, Lin F, Yuan W, Sun G, Tao Z. GhABF2, a bZIP transcription factor, confers drought and salinity tolerance in cotton (Gossypium hirsutum L.). Sci Rep. 2016;6:35040.

19. Cuartero J, Bolarin M, Asins M, Moreno V. Increasing salt tolerance in the tomato. J Exp Bot. 2006;57(5):1045-58.

20. Li D, Fu F, Zhang H, Song F. Genome-wide systematic characterization of the bZIP transcriptional factor family in tomato (Solanum lycopersicum L.). BMC Genomics. 2015:16(1):771.

21. Bastías A, Yañez M, Osorio S, Arbona V, Gómezcadenas A, Fernie AR, Casaretto JA. The transcription factor AREB1 regulates primary metabolic pathways in tomato fruits. J Exp Bot. 2014;65(9):2351-63.

22. Orellana S, Yanez M, Espinoza A, Verdugo I, Gonzalez E, RUIZ-LARA S, Casaretto JA. The transcription factor SIAREB1 confers drought, salt stress tolerance and regulates biotic and abiotic stress-related genes in tomato. Plant Cell Environ. 2010;33(12):2191-208

23. Hsieh TH, Li CW, Su RC, Cheng CP, Tsai YC, Chan MT. A tomato bZIP transcription factor, SIAREB, is involved in water deficit and salt stress response. Planta. 2010;231(6):1459-73.

24. Sell S, Hehl R. Functional dissection of a small anaerobically induced bZIP transcription factor from tomato. Eur J Biochem. 2004:271(22):4534-44.

25. Abe M, Kobayashi $Y$, Yamamoto S, Daimon Y, Yamaguchi A, Ikeda Y, Ichinoki H, Notaguchi M, Goto K, Araki T. FD, a bZIP protein mediating signals from the floral pathway integrator FT at the shoot apex. Science. 2005;309(5737):1052-6.

26. Seong ES, Kwon SS, Ghimire BK, Yu CY, Cho DH, Lim JD, Kim KS, Heo K, Lim ES, Chung IM. LebZIP2 induced by salt and drought stress and transient overexpression by Agrobacterium. BMB Rep. 2008;41(10):693-8.

27. Stanković B, Vian A, Henryvian C, Davies E. Molecular cloning and characterization of a tomato CDNA encoding a systemically woundinducible bZIP DNA-binding protein. Planta. 2000;212(1):60-6.

28. Ji K, Kai W, Zhao B, Sun Y, Yuan B, Dai S, Li Q, Chen P, Wang Y, Pei Y. SINCED1 and SICYP707A2: key genes involved in ABA metabolism during tomato fruit ripening. J Exp Bot. 2014;65(18):5243-55.

29. Danquah A, Zelicourt AD, Colcombet J, Hirt H. The role of ABA and MAPK signaling pathways in plant abiotic stress responses. Biotechnol Adv. 2014 32(1):40-52.

30. Chen P, Sun Y-F, Kai W-B, Liang B, Zhang Y-S, Zhai X-W, Jiang L, Du Y-W, Leng $P$. Interactions of ABA signaling core components (SIPYLS, SIPP2CS, and SISnRK2s) in tomato (Solanum lycopersicon). J Plant Physiol. 2016;205:67-74.

31. Zhao P, Li Q, Li J, Wang L, Ren Z. Genome-wide identification and characterization of R2R3MYB family in Solanum lycopersicum. Mol Gen Genomics. 2014;289(6):1183-207.

32. Gawehns FKK. Function and targets of Fusarium oxysporum effectors. In Chapter 5. University of Amsterdam; 2014. p. 98-126.

33. Etalo DW. An integrated approach involving metabolomics and transcriptomics for a system-wide understanding of the interaction between tomato and Cladosporium fulvum. In Chapter 3. Wageningen University; 2014. p. 101-48.

34. Nakano T, Fujisawa M, Shima Y, Ito Y. Expression profiling of tomato preabscission pedicels provides insights into abscission zone properties including competence to respond to abscission signals. BMC Plant Biol. 2013;13(1):40.

35. Jie W, Pan C, Yan W, Lei Y, Jian W, Chen L, Tao Z, Gang L. Genome-wide identification of MAPKK and MAPKKK gene families in tomato and transcriptional profiling analysis during development and stress response. PLoS One. 2014;9(7):e103032.

36. Yu C, Cai X, Ye Z, Li H. Genome-wide identification and expression profiling analysis of trihelix gene family in tomato. Biochem Biophys Res Commun. 2015;468(4):653-9.

37. Liu L, Liu H, Li S, Zhang X, Zhang M, Zhu N, Dufresne CP, Chen S, Wang Q. Regulation of BZR1 in fruit ripening revealed by $\mathrm{ITRAQ}$ proteomics analysis. Sci Rep. 2016;6:33635.

38. Dietrich K, Weltmeier F, Ehlert A, Weiste C, Stahl M, Harter K, Dröge-Laser W. Heterodimers of the Arabidopsis transcription factors bZIP1 and bZIP53 reprogram amino acid metabolism during low energy stress. Plant Cell. 2011:23(1):381

39. Zhu JK. Salt and drought stress signal transduction in plants. Annu Rev Plant Biol. 2002:53:247.

40. Hong Y, Zhang H, Huang L, Li D, Song F. Overexpression of a stressresponsive NAC transcription factor gene ONACO22 improves drought and salt tolerance in Rice. Front Plant Sci. 2016;7(e0116646):4.
41. Xu X-y, Shi G-X, Wang J, Kang Y-N. Copper-induced oxidative stress in Alternanthera philoxeroides callus. Plant Cell Tissue Organ Cult. 2011;106(2):243-51.

42. Gill SS, Tuteja N. Reactive oxygen species and antioxidant machinery in abiotic stress tolerance in crop plants. Plant Physiol Biochem. 2010;48(12):909-30.

43. Matés JM. Effects of antioxidant enzymes in the molecular control of reactive oxygen species toxicology. Toxicology. 2000;153(1-3):83-104.

44. Roy R, Purty RS, Agrawal V, Gupta SC. Transformation of tomato cultivar 'Pusa ruby' with bspA gene from Populus tremula for drought tolerance. Plant Cell Tissue Organ Cult. 2006;84(1):56-68.

45. Mittler R. Oxidative stress, antioxidants and stress tolerance. Trends Plant Sci. 2002;7(9):405-10.

46. Zhang J, Jia W, Yang J, Ismail AM. Role of ABA in integrating plant responses to drought and salt stresses. Field Crop Res. 2006:97(1):111-9.

47. Iuchi S, Kobayashi M, Taji T, Naramoto M, Seki M, Kato T, Tabata S, Kakubari Y, Yamaguchi-Shinozaki K, Shinozaki K. Regulation of drought tolerance by gene manipulation of 9-cis-epoxycarotenoid dioxygenase, a key enzyme in abscisic acid biosynthesis in Arabidopsis. Plant J Cell Mol Biol. 2001;27(4):325-33.

48. Tu M, Wang X, Feng $T$, Sun X, Wang Y, Huang L, Gao M, Wang Y, Wang X. Expression of a grape (Vitis vinifera) bZIP transcription factor, VlbZIP36, in Arabidopsis thaliana confers tolerance of drought stress during seed germination and seedling establishment. Plant Sci. 2016;252:311-23.

49. Xiong L, Schumaker KS, Zhu JK. Cell signaling during cold, drought, and salt stress. Plant Cell. 2002;14(Suppl):S165-83.

50. Zhu M, Chen G, Zhou S, Tu Y, Wang Y, Dong T, Hu Z. A new tomato NAC (NAM) ATAF1/2/CUC2) transcription factor, SINAC4, functions as a positive regulator of fruit ripening and carotenoid accumulation. Plant Cell Physiol. 2014;55(1):119-35.

51. Zhu M, Hu Z, Zhou S, Wang L, Dong T, Pan Y, Chen G. Molecular characterization of six tissue-specific or stress-inducible genes of NAC transcription factor family in tomato (Solanum lycopersicum). J Plant Growth Regul. 2014;33(4):730-44.

52. Expósito-Rodríguez M, Borges AA, Borges-Pérez A, Pérez JA. Selection of internal control genes for quantitative real-time RT-PCR studies during tomato development process. BMC Plant Biol. 2008:8(1):131.

53. Nicot N, Hausman J-F, Hoffmann L, Evers D. Housekeeping gene selection for real-time RT-PCR normalization in potato during biotic and abiotic stress. J Exp Bot. 2005;56(421):2907-14.

54. Zhang X, Ja TDS, Duan J, Deng R, Xu X, Ma G. Endogenous hormone levels and anatomical characters of haustoria in Santalum album L. seedlings before and after attachment to the host. J Plant Physiol. 2012;169(9):859-66.

55. Zhu M, Chen G, Dong T, Wang L, Zhang J, Zhao Z, Hu Z. SIDEAD31, a putative DEAD-box RNA helicase gene, regulates salt and drought tolerance and stress-related genes in tomato. PLoS One. 2015;10(8):e0133849.

56. Góth L. A simple method for determination of serum catalase activity and revision of reference range. Clin Chim Acta. 1991;196(2-3):143-51.

57. Zhang G, Chen M, Li L, Xu Z, Chen X, Guo J, Ma Y. Overexpression of the soybean GmERF3 gene, an AP2/ERF type transcription factor for increased tolerances to salt, drought, and diseases in transgenic tobacco. J Exp Bot. 2009:60(13):3781.

58. Kim D, Pertea G, Trapnell C, Pimentel H, Kelley R, Salzberg SL. TopHat2: accurate alignment of transcriptomes in the presence of insertions, deletions and gene fusions. Genome Biol. 2013;14(4):R36.

59. Pertea M, Pertea GM, Antonescu CM, Chang TC, Mendell JT, Salzberg SL. StringTie enables improved reconstruction of a transcriptome from RNA-seq reads. Nat Biotechnol. 2015;33(3):290-5.

60. Le DT, Nishiyama R, Watanabe Y, Mochida K, Yamaguchi-Shinozaki K, Shinozaki K, Tran L-SP. Genome-wide survey and expression analysis of the plant-specific NAC transcription factor family in soybean during development and dehydration stress. DNA Res. 2011;18(4):263-76. 